\title{
Saúde Bucal em crianças hospitalizadas com câncer: conhecimentos e práticas dos cuidadores
}

\author{
Oral health in children with cancer: knowledge and practices of caregivers \\ Salud bucal en niños hospitalizados con cáncer: conocimientos y prácticas de los cuidadores
}

Recebido: 11/08/2021 | Revisado: 22/08/2021 | Aceito: 24/08/2021 | Publicado: 25/08/2021

Adrielly Carvalho Guedes

ORCID: https://orcid.org/0000-0003-1627-2484 Universidade Federal do Amazonas, Brasil

E-mail: adriellyguedes@gmail.com

Valéria Melo Lima

ORCID: https://orcid.org/0000-0001-9762-0318 Universidade Federal do Amazonas, Brasil

E-mail:valeria_lima@outlook.com

Sara Lais Silva Mendonça

ORCID: https://orcid.org/0000-0001-9558-6136 Universidade Federal do Amazonas, Brasil E-mail: lais21sara@gmail.com

Simone Assayag Hanan

ORCID: https://orcid.org/0000-0002-3415-8557

Universidade Federal do Amazonas, Brasil

E-mail: simonehanan@yahoo.com.br

Ary de Oliveira Alves Filho

ORCID: https://orcid.org/0000-0003-4143-289X

Universidade Federal do Amazonas, Brasil

E-mail: aryalvesfilho@gmail.com

Jardel dos Santos Silva

ORCID: https://orcid.org/0000-0002-5248-1075

Universidade Federal do Amazonas, Brasil

E-mail: jardel.santos17@hotmail.com

Rachid Pinto Zacarias Filho

ORCID: https://orcid.org/0000-0003-0720-9328

Universidade do Estado do Amazonas, Brasil

E-mail: rachidfilho@hotmail.com

Aida Renée Assayag Hanan

ORCID: https://orcid.org/0000-0001-6430-1791

Universidade Federal do Amazonas, Brasil

E-mail: aidahanan@hotmail.com

Pollyanna Oliveira Medina

ORCID: https://orcid.org/0000-0002-1646-0277

Universidade Federal do Amazonas, Brasil E-mail: pmedina@ufam.edu.br

\begin{abstract}
Resumo
Objetivos: Avaliar o conhecimento de cuidadores e suas práticas de saúde bucal em crianças com câncer hospitalizadas, além de relatar as principais complicações bucais decorrentes do tratamento oncológico. Metodologia: Estudo transversal com 34 cuidadores de crianças com câncer hospitalizadas na Fundação de Controle de Oncologia do Estado do Amazonas (FCECON), na faixa etária de 0 a12 anos de idade. Resultados: Dos cuidadores, 70,6\% eram do gênero feminino, predominantemente mães (70,6\%), na faixa etária de 31 a 40 anos (52,9\%), solteiros $(52,9 \%)$ e desempregados $(73,5 \%)$. Apenas 32,8\% receberam instruções de higiene oral, sendo o Cirurgião-Dentista a fonte destas em somente $32,4 \%$ dos casos. A maioria dos cuidadores realizava a higiene bucal de seus filhos três vezes ao dia (76,5\%), usando apenas escova dental e dentifrício (61,8\%). Apenas 23,5\% das crianças relataram desconforto na cavidade bucal e $97,1 \%$ dos cuidadores relataram ser importante a inclusão do Cirurgião-Dentista na equipe de saúde. Dentre as manifestações bucais pós-tratamento oncológico, as mais prevalentes foram a alteração no paladar (32,4\%) e a secura bucal $(26,5 \%)$. O período de internação foi de até 7 dias $(61,8 \%)$ e o tempo de tratamento superior a um ano $(34,4 \%)$. Conclusão: Constatou-se falta de informação dos cuidadores sobre as práticas de higiene bucal e a dificuldade em entender a importância da saúde bucal para manutenção da saúde sistêmica, apesar de relatarem a ocorrência frequente de disgeusia e secura bucal, o que pode influenciar negativamente no sucesso do tratamento médico e até mesmo levar o paciente a óbito.
\end{abstract}

Palavras-chave: Neoplasias; Criança; Cuidadores; Saúde bucal; Conhecimento. 


\begin{abstract}
Objectives: To assess the knowledge of caregivers and their oral health practices in hospitalized children with cancer, in addition to reporting the main oral complications resulting from cancer treatment. Methodology: Cross-sectional study with 34 caregivers of children with cancer hospitalized at the Oncology Control Foundation of the State of Amazonas (FCECON), aged 0-12 years. Results: Of the caregivers, 70.6\% were female, predominantly mothers $(70.6 \%)$, aged between 31 and 40 years $(52.9 \%)$, single $(52.9 \%)$ and unemployed $(73,5 \%)$. Only $32.8 \%$ received oral hygiene instructions, with the dentist being the source of these in only $32.4 \%$ of cases. Most caregivers performed their children's oral hygiene three times a day (76.5\%), using only a toothbrush and toothpaste (61.8\%). Only $23.5 \%$ of children reported discomfort in the oral cavity and $97.1 \%$ of caregivers reported that the inclusion of the Dental Surgeon in the health team was important. Among the oral manifestations after cancer treatment, the most prevalent were changes in taste $(32.4 \%)$ and dry mouth $(26.5 \%)$. The hospital stay was up to 7 days $(61.8 \%)$ and the treatment time was over one year (34.4\%). Conclusion: There was a lack of information from caregivers about oral hygiene practices and the difficulty in understanding the importance of oral health for the maintenance of systemic health, despite reporting the frequent occurrence of dysgeusia and oral dryness, which can negatively influence the success of medical treatment and even lead the patient to death.
\end{abstract}

Keywords: Neoplasms; Child; Caregivers; Oral health; Knowledge.

\title{
Resumen
}

Objetivos: Evaluar el conocimiento de los cuidadores y sus prácticas de salud bucal en niños hospitalizados con cáncer, además de informar de las principales complicaciones bucales derivadas del tratamiento oncológico. Metodología: Estudio transversal con 34 cuidadores de niños con cáncer hospitalizados en la Fundación de Control Oncológico del Estado de Amazonas (FCECON), de 0 a 12 años. Resultados: De los cuidadores, el 70,6\% eran mujeres, predominantemente madres $(70,6 \%)$, con edades entre 31 y 40 años $(52,9 \%)$, solteras $(52,9 \%)$ y desempleadas (73,5\%). Solo el 32,8\% recibió instrucciones de higiene bucal, siendo el odontólogo la fuente de estas en solo el 32,4\% de los casos. La mayoría de los cuidadores realizaba la higiene bucal de sus hijos tres veces al día (76,5\%), utilizando solo cepillo de dientes y pasta de dientes (61,8\%). Solo el 23,5\% de los niños refirió malestar en la cavidad bucal y el 97,1\% de los cuidadores refirió que la inclusión del Cirujano Dentista en el equipo de salud fue importante. Entre las manifestaciones orales posteriores al tratamiento del cáncer, las más prevalentes fueron alteraciones del gusto $(32,4 \%)$ y sequedad de boca (26,5\%). La estancia hospitalaria fue de hasta 7 días $(61,8 \%)$ y el tiempo de tratamiento fue superior a un año $(34,4 \%)$. Conclusión: hubo una falta de información por parte de los cuidadores sobre las prácticas de higiene bucal y la dificultad para comprender la importancia de la salud bucal para el mantenimiento de la salud sistémica, a pesar de reportar la ocurrencia frecuente de disgeusia y sequedad bucal, las cuales pueden influir negativamente en el éxito de los tratamientos médicos. tratamiento e incluso llevar al paciente a la muerte.

Palabras clave: Neoplasias; Niño; Cuidadores; Salud bucal; Conocimiento.

\section{Introdução}

O câncer infantojuvenil compreende um grupo de várias doenças, de origem predominantemente embrionária, caracterizadas pelo crescimento desordenado de células indiferenciadas que possuem a capacidade de se disseminar entre os tecidos e órgãos de qualquer local do organismo, o que geralmente proporciona melhor resposta aos tratamentos atuais, apesar do crescimento rápido e da maior agressividade da doença. No Brasil, o câncer já representa $8 \%$ do total de óbitos por doença entre crianças e adolescentes de 1 a 19 anos e há a estimativa que para cada ano do triênio 2020/2022, sejam diagnosticados no Brasil 8.460 novos casos de câncer infantojuvenis, sendo 630 no Estado do Amazonas (Brasil, 2020).

Dentre os tumores mais prevalentes na infância e na adolescência, destacam-se as leucemias, seguidas dos que atingem o sistema nervoso central e dos linfomas (Brasil, 2020). Na leucemia, as alterações patológicas na cavidade bucal ocorrem frequentemente, como a presença de úlceras bucais e o sangramento gengival.

O tratamento do câncer infantojuvenil tem avançado significativamente, em virtude das técnicas de diagnóstico precoce e da evolução dos métodos terapêuticos (Brasil, 2008). A terapia antineoplásica, apesar de trazer consigo a possibilidade da cura, traz também efeitos colaterais severos, inclusive na cavidade bucal, onde várias lesões e/ou alterações bucais podem ser observadas, como a mucosite, a candidíase, a disgeusia e a xerostomia, tanto quanto a doença em si. A xerostomia é caracterizada pela secura bucal devido à insuficiente produção de saliva, denominada de hipossalivação, sendo uma alteração frequente nos pacientes que fazem tratamento antioneoplásico (Lopes, et al., 2012). A alteração do paladar 
causada pela quimioterapia, uma das sequelas mais encontradas, geralmente desaparece após algumas semanas (Volpato, et al., 2014). Manifestações como a mucosite e a xerostomia afetam atividades simples como a alimentação e a comunicação, além de que podem predispor o indivíduo a infecções bucais e, consequentemente, interferir no tratamento do câncer (Velten, et al., 2017).

Durante o tratamento, a criança e a família passam por mudanças significativas no dia a dia; normalmente, a criança ficará longe da escola e amigos, apresentará algumas limitações físicas, prejudicando sua qualidade de vida, e os pais precisam se adaptar à nova condição de saúde que seu filho apresenta (Benedetti, et al., 2014).

Segundo Sommerfeld, et al. (2011), o primeiro impacto é o diagnóstico, o que suscita várias emoções na criança e na família, seguido de longo e sofrido tratamento, com diversas reações, causando constrangimentos, mudanças na aparência física e baixa resistência biológica, afastamento do meio social e de atividades físicas, separação do lar e mudanças na rotina diária. Além disso, são submetidas a uma série de procedimentos que acentuam a dor e o sofrimento.

O tratamento odontológico dos pacientes submetidos ao tratamento oncológico torna-se indispensável, em razão da imunossupressão, a fim de minimizar o risco do aparecimento, ou ainda, a detecção precoce de lesões orais decorrentes da doença e/ou do seu tratamento. Desta forma, o cuidado com a promoção e manutenção da saúde, tanto geral, quanto bucal, torna-se imprescindível e deve envolver a abordagem de equipes multidisciplinares, em que o Cirurgião-Dentista esteja inserido, na tentativa de auxiliar na melhoria do quadro durante a hospitalização, sendo essencial incluir no cuidado à criança o seu cuidador (Ballestreri, et al., 2016; Ponte, et al., 2019).

Tendo em vista que a presença de higiene bucal deficiente ou a pré-existência de focos infecciosos elevam o risco de infecção bucal durante o tratamento antineoplásico, aumentando também o risco de complicações sistêmicas e o tempo de hospitalização, além de causar prejuízos na qualidade de vida e no bem-estar da criança, o presente estudo teve como objetivo avaliar o conhecimento de cuidadores e suas práticas de saúde bucal em crianças com câncer de até 12 anos de idade, hospitalizadas na Fundação Centro de Controle de Oncologia do Estado do Amazonas (FCECON), além de relatar as principais complicações bucais decorrentes do tratamento oncológico, sob a ótica da percepção do cuidador.

\section{Metodologia}

Trata-se de um estudo do tipo observacional transversal para avaliar o conhecimento dos cuidadores de crianças em tratamento antineoplásico na FCECON, localizada no município de Manaus-AM. Fundada em 1974, como Centro de Oncologia (CECON), a unidade hospitalar foi transformada em 1989, em Fundação, reunindo diversas especialidades médicas e os principais tratamentos oncológicos, fato que consolidou a instituição como referência no diagnóstico e tratamento do câncer em toda a Amazônia Ocidental (Amazonas, Acre, Rondônia e Roraima). Além do renomado corpo clínico, a Fundação também se destaca pelas ações desenvolvidas nas áreas de Prevenção e Ensino e Pesquisa, as quais foram ampliadas significativamente, nos últimos anos, com o aumento do número de campanhas de combate à doença e pesquisas, fortalecendo a área científica no Estado.

A população inicial deste estudo foi constituída de uma amostra não probabilística representada por todos os 62 cuidadores (pais/responsáveis) de crianças de até 12 anos de idade, hospitalizados na FCECON, em tratamento antineoplásico, de janeiro a maio de 2019. Os critérios de inclusão foram: cuidadores maiores de 18 anos de idade que acompanhavam crianças com câncer, de ambos os sexos, na faixa etária de 0 a 12 anos de idade, hospitalizadas na FCECON. Foram excluídos aqueles que se recusaram a assinar o Termo de Consentimento Livre e Esclarecido ou a preencher o questionário, aqueles impossibilitados de ler ou escrever e, ainda, aqueles que não responderam a todas as perguntas do questionário.

Após a autorização da instituição participante, os indivíduos foram convidados a participar do estudo, aleatoriamente, através do Termo de Consentimento Livre e Esclarecido, com aprovação do CEP. Em concordância com a Resolução 466/12 
do Conselho Nacional de Saúde, principalmente no que concerne à autorização dos participantes, sigilo e confidencialidade, após a assinatura do Termo, os cuidadores que obedeceram aos critérios de inclusão e exclusão foram incluídos no estudo. A pesquisa foi realizada dentro das normas exigidas e aprovada pelo Comitê de Ética em Pesquisa com seres humanos sob o número do protocolo: 00724818.6.0000.5020.

O instrumento da coleta de dados foi um questionário, aplicado aos cuidadores, em uma sala reservada próxima aos leitos pediátricos, por duas pesquisadoras previamente calibradas, que não possuíam nenhum vínculo com as famílias participantes, contendo questões abertas e de múltipla escolha acerca do perfil sociodemográfico dos cuidadores e sobre o conhecimento e a prática de saúde bucal em crianças acometidas por câncer (adaptado de Barbosa, et al., 2010), que esses indivíduos acompanhavam.

Os dados foram apresentados por meio de gráficos e tabelas, onde foram calculadas as frequências absolutas simples e relativas para os dados categóricos (gênero, idade, grau de parentesco, estado civil, situação profissional, além das informações relativas ao conhecimento dos cuidadores acerca da saúde bucal de seus filhos). $\mathrm{Na}$ análise dos dados categorizados, aplicou-se o teste do qui-quadrado de Pearson, sendo que na impossibilidade de aplicá-lo, em razão de restrições em seu uso (se $20<\mathrm{n}<$ 40, só pode ser aplicado se nenhuma frequência esperada for menor que 1), foi aplicado o teste exato de Fisher. O software utilizado na análise dos dados foi o programa Epi Info 7.2.2 para Windows, com nível de significância de 5\%.

\section{Resultados}

Dos 62 indivíduos convidados, 49 cuidadores aceitaram participar, porém apenas 34 atenderam aos critérios de inclusão e exclusão do estudo. A Tabela 1 expressa os dados sociodemográficos dos cuidadores de pacientes pediátricos em tratamento antineoplásico na FCECON, onde quanto ao grau de parentesco, observou-se que apenas um $(2,9 \%)$ dos cuidadores era o avô da criança hospitalizada. 
Tabela 1. Distribuição segundo os dados sociodemográficos dos cuidadores de pacientes pediátricos em tratamento antineoplásico na Fundação Centro de Controle de Oncologia do Estado do Amazonas (FCECON). Manaus - AM.

\begin{tabular}{|c|c|c|}
\hline Variáveis $(n=34)$ & $f_{i}$ & $\%$ \\
\hline \multicolumn{3}{|l|}{ Gênero } \\
\hline Feminino & 24 & 70,6 \\
\hline Masculino & 10 & 29,4 \\
\hline \multicolumn{3}{|l|}{ Idade } \\
\hline$<20$ anos & 2 & 5,9 \\
\hline 20 a 30 anos & 7 & 20,6 \\
\hline 31 a 40 anos & 18 & 52,9 \\
\hline$>40$ anos & 7 & 20,6 \\
\hline \multicolumn{3}{|c|}{ Média Idade \pm DP $(33,7 \pm 7,8$ anos $)$} \\
\hline \multicolumn{3}{|l|}{ Grau de parentesco } \\
\hline Mãe & 24 & 70,6 \\
\hline Pai & 9 & 26,5 \\
\hline Outro & 1 & 2,9 \\
\hline \multicolumn{3}{|l|}{ Estado Civil } \\
\hline Casado & 16 & 47,1 \\
\hline Solteiro & 18 & 52,9 \\
\hline \multicolumn{3}{|c|}{ Situação profissional } \\
\hline Empregado & 5 & 14,7 \\
\hline Desempregado & 25 & 73,5 \\
\hline Aposentado & 1 & 2,9 \\
\hline Não trabalha & 3 & 8,8 \\
\hline
\end{tabular}

$\mathrm{fi}=$ frequência absoluta simples. Fonte: Autores (2021).

Foram analisados os resultados quanto aos conhecimentos e práticas em saúde bucal dos cuidadores das crianças com câncer hospitalizadas, esquematizados na Tabela 2 , na qual a maior parte dos cuidadores $(61,8 \%)$ relatou que as instruções foram dadas por outros (mídia, amigos, vizinhos, etc) e não pela equipe de profissionais da FCECON. 
Tabela 2. Distribuição segundo os conhecimentos e práticas de saúde bucal dos cuidadores dos pacientes pediátricos em tratamento antineoplásico na Fundação Centro de Controle de Oncologia do Estado do Amazonas (FCECON). Manaus - AM.

\begin{tabular}{|c|c|c|}
\hline Variáveis $(n=34)$ & $f_{i}$ & $\%$ \\
\hline Receberam instruções de higiene bucal & 13 & 38,2 \\
\hline \multicolumn{3}{|l|}{ Quem forneceu as informações sobre higiene bucal } \\
\hline Equipe médica FCECON & 1 & 2,9 \\
\hline Cirurgião-dentista FCECON & 11 & 32,4 \\
\hline Equipe de Enfermagem FCECON & 1 & 2,9 \\
\hline Outros & 21 & 61,8 \\
\hline Realiza a higiene bucal da criança & 26 & 76,5 \\
\hline \multicolumn{3}{|l|}{ Frequência é realizada a higiene bucal } \\
\hline Duas vezes ao dia & 7 & 20,6 \\
\hline Três vezes ao dia & 26 & 76,5 \\
\hline Mais de três vezes ao dia & 1 & 2,9 \\
\hline \multicolumn{3}{|l|}{ O que realiza ou indica para a higiene bucal } \\
\hline Escova, dentifrício & 21 & 61,8 \\
\hline Escova, dentifrício e fio dental & 9 & 26,5 \\
\hline Escova, dentifrício e clorexidina & 2 & 5,9 \\
\hline Escova, dentifrício fio dental e clorexidina & 2 & 5,9 \\
\hline Criança relata desconforto na cavidade bucal & 8 & 23,5 \\
\hline Criança é encaminhada para o cirurgião-dentista & 11 & 32,4 \\
\hline Acha importante ter um cirurgião-dentista na equipe & 33 & 97,1 \\
\hline
\end{tabular}

$$
\text { fi = frequência absoluta simples. Fonte: Autores (2021). }
$$

Quando associados os conhecimentos e práticas em saúde bucal dos cuidadores ao grau de parentesco, observou-se relação estatisticamente significativa apenas com o executor da prática de escovação, sendo esta realizada majoritariamente pelas mães $(\mathrm{p}=0,031)$.

Dados sobre o conhecimento dos cuidadores acerca das manifestações cínicas e bucais pós-tratamento antineoplásico estão descritos na Tabela 3. Não houve associação significativa entre o grau de parentesco e o relato dos cuidadores quanto às manifestações clínicas pós-tratamento antineoplásico ( $>>0,05)$. 
Tabela 3. Distribuição segundo os conhecimentos dos cuidadores acerca das manifestações clínicas após tratamento dos pacientes em tratamento antineoplásico na Fundação Centro de Controle de Oncologia do Estado do Amazonas (FCECON). Manaus - AM.

\begin{tabular}{lcc}
\hline \hline Variáveis $(n=34)$ & $f_{i}$ & $\%$ \\
\hline Manifestações clínicas após tratamento & & \\
Nenhuma & 4 & 11,8 \\
Enjoos & 23 & 67,6 \\
Vômitos & 22 & 64,7 \\
Ausência de paladar & 11 & 32,4 \\
Boca seca & 9 & 26,5 \\
Dor na mucosa & 8 & 23,5 \\
Dor de dente & 8 & 23,5 \\
Ardência & 3 & 8,8 \\
\hline \hline
\end{tabular}

$\mathrm{fi}=$ frequência absoluta simples. Fonte: Autores (2021).

Os tempos de tratamento e de internação, assim como os tipos de câncer mais prevalentes dentre as crianças investigadas estão demonstrados na Tabela 4.

Tabela 4. Distribuição segundo os dados clínicos dos pacientes pediátricos. em tratamento antineoplásico na Fundação Centro de Controle de Oncologia do Estado do Amazonas (FCECON). Manaus - AM.

\begin{tabular}{lcc}
\hline \hline Variáveis $(n=34)$ & $f_{i}$ & $\%$ \\
\hline Tempo de tratamento & & \\
$\leq 30$ dias & 5 & 15,6 \\
$30--\mid 180$ dias & 8 & 25,0 \\
$180--\mid 365$ dias & 8 & 25,0 \\
$>365$ dias & 11 & 34,4 \\
Tempo de internação & & \\
$\leq 7$ dias & 21 & 61,8 \\
$>7$ dias & 13 & 38,2 \\
Diagnóstico & & \\
Neuroblastoma & 5 & 14,7 \\
Osteossarcoma & 6 & 17,6 \\
Outros & 23 & 67,7 \\
\hline \hline
\end{tabular}

fi $=$ frequência absoluta simples. Fonte: Autores (2021).

\section{Discussão}

No presente estudo, a maioria dos cuidadores foram as mães, que usualmente dedicam suas vidas aos cuidados dos filhos; durante a condução do tratamento oncológico, muitas até abandonaram seus empregos para dedicarem-se, exclusivamente, a esses cuidados, em concordância com os estudos de Benedetti, et al. (2014), Ballestreri, et al. (2016), Ponte, 
et al. (2019) e de Andrade, et al. (2020), em que a mãe tem sido apontada como cuidadora principal de crianças. Essa situação é explicada tendo por base o elo estabelecido entre mãe-filho desde a gestação até a infância da criança, expresso posteriormente pela dependência e referência da criança pela figura materna. Tal elo mostra-se essencialmente importante na transmissão de hábitos de higiene bucal e dietéticos da mãe para o filho (Ballestreri, et al., 2016).

Outro resultado a ser ressaltado foi a média de idade do cuidador, 33,7 anos, mas com desvio padrão de 7,8. Dados do Instituto Brasileiro de Geografia e Estatística (Brasil, 2019) apontam que do total de nascidos vivos em 2019, 48,3\% das mães tinham entre 20 e 29 anos e 37,5\% tinham mais de 30 anos, embora no presente estudo haja o desconhecimento do fato da criança internada ser de uma primeira gestação, além de não ter sido relacionada a idade da criança à da mãe.

Em um estudo realizado por Huesca, et al. (2018), foi observado que um dos primeiros impactos do tratamento antineoplásico para a família é a perda ou diminuição da renda. Em nosso estudo, grande parte dos cuidadores entrevistados encontravam-se desempregados, talvez pela necessidade de se dedicarem exclusivamente ao tratamento da criança doente, pois muitos deles relataram ter pedido demissão ou terem sido demitidos de seus empregos, em razão do absenteísmo laboral, logo após o diagnóstico da doença (Pinheiro, et al., 2021).

O diagnóstico de um câncer pode determinar um grande impacto emocional familiar, conduzindo a diferentes sentimentos e emoções, e quando o paciente é uma criança, ou um adolescente, esse abalo pode ser ainda maior. Muitos pais não conseguem suportar esta abrupta carga emocional, não só pelo diagnóstico da doença, mas também pelo tratamento oncológico, que irá exigir bastante dos familiares mais próximos. Não há aqui como estabelecer uma relação entre o diagnóstico do câncer infantil e o estado civil das entrevistadas, uma vez que esse não foi o foco da pesquisa. No entanto, Finelly, et al. (2015) apontam que o apoio do companheiro é um fator importante que ajuda no restabelecimento do estado de saúde mental do outro cuidador. Sendo assim, o fato de mais da metade das mães estarem solteiras em nosso estudo, sem ter com quem compartilhar os cuidados diários com a criança, possivelmente poderia fazê-las sentir ainda mais desamparadas em função da ausência de um companheiro que lhes fornecesse apoio e as ajudasse de forma mais efetiva durante o diagnóstico e o tratamento do câncer infantil.

A higiene bucal é essencial em crianças com câncer, por diminuir o risco do aparecimento de complicações bucais, já que são pacientes imunossuprimidos e mais vulneráveis a infecções (Ponte, et al., 2019). A prática do controle mecânico do biofilme dental não pode ser negligenciada, tendo em vista que a presença deste favoreceria a ocorrência de doença periodontal e de sangramento gengival espontâneo, o qual poderia se exacerbar em pacientes com plaquetopenia e mucosite (Peres, et al., 2013). Sabe-se que o tratamento oncológico exige ótimas condições de saúde bucal, uma vez que patologias bucais préexistentes podem ser agravadas, podendo provocar inclusive a sua interrupção (Sommerfeld, et al., 2011).

A FCECON apresenta como um dos protocolos de atendimento uma avaliação odontológica prévia ao tratamento antineoplásico, onde o Cirurgião-Dentista realiza a visita aos pacientes que estão internados quando é solicitado pela equipe de saúde; porém, entre os cuidadores entrevistados, apenas poucos relataram que as crianças foram avaliadas pelo CirurgiãoDentista, semelhante ao resultado encontrado por Floriano, et al.(2017), no qual a grande maioria não passou pela avaliação odontológica prévia. No estudo de Ponte, et al. (2019), algumas entrevistadas também afirmaram que as suas crianças nunca foram encaminhadas ao dentista, sendo comum o discurso de que as crianças só poderiam ser encaminhadas para atendimento odontológico quando finalizassem o tratamento antineoplásico. Tal fato denota a dificuldade na relação médico-dentista, demonstrando a necessidade de revisão do protocolo existente, que assegure que tal interação seja estabelecida de forma harmoniosa e segura.

Quanto à higiene bucal das crianças, os cuidadores relataram, na maioria dos casos, que não receberam instruções de higiene bucal da equipe de saúde da instituição e sim de outros profissionais em um outro momento, anterior ao diagnóstico de 
câncer, e que utilizavam como instrumentos apenas a escova e o dentifrício, semelhante ao encontrado nos estudos de Rottini, et al. (2019) e de Ponte, et al. (2019).

A maioria dos cuidadores fazia o acompanhamento da higiene bucal de suas crianças, realizando a escovação dentária três vezes ao dia, predominantemente executada pelas mães $(\mathrm{p}=0,031)$, reforçando mais uma vez a inserção da figura da mãe, desde o início da infância da criança, no cuidado diário de seus filhos. Tal prática mostra-se de extrema importância, principalmente em crianças debilitadas, onde há uma tendência de maior permissividade a fatores não favoráveis e o descuido com a saúde bucal em momentos de agravamento do quadro (Ponte, et al., 2019). A frequência da escovação dentária usada pela população em geral pode variar; entretanto, a mais relatada em pesquisas usando entrevistas e questionários é de três vezes ao dia. É provável que o conhecimento sobre a frequência de escovação adequada tenha levado a um viés de resposta (convenção social), superestimando a frequência real de escovação (Grado, et al., 2021). Uma meta-análise confirmou que o aumento da frequência de escovação está associado a uma baixa incidência de cárie e ao menor incremento de novas lesões, especialmente na dentição decídua (Kumar, et al., 2016). A falta de higiene bucal pode gerar implicações negativas na qualidade de vida de pacientes oncológicos, tendo potencial para refletir em vários âmbitos da saúde sistêmica do indivíduo (Gandhi et al., 2017). A Academia Americana de Odontopediatria (2020), a Associação Internacional de Odontopediatria (2020) e a Associação Brasileira de Odontopediatria (2020) recomendam a escovação dentária com o uso de dentifrício fluoretado na concentração mínima de $1.000 \mathrm{ppm}$ duas vezes ao dia para todas as crianças.

Embora na literatura científica existam vários relatos de manifestações bucais em crianças com câncer (Peres, et al., 2013; Velten, et al, 2017; Rottini, et al., 2019), alguns investigados, quando questionados da presença de incômodo ou desconforto na boca, responderam que a criança não manifestava desconforto na cavidade bucal, corroborando com os achados de Ponte, et al.(2019). Tal fato provavelmente pode encontrar-se relacionado à prática diária da higiene bucal das crianças por parte dos cuidadores, o que contribuiria para minimizar os riscos do surgimento de complicações bucais em pacientes oncológicos.

Quando questionados se achavam importante ter um Cirurgião-Dentista na equipe de saúde, quase a totalidade dos cuidadores afirmaram que sim, resultado semelhante ao reportado por Barbosa, et al. (2010), onde todos os cuidadores investigados responderam achar importante a presença do profissional na equipe médica. A presença do Cirurgião-Dentista, em equipes multiprofissionais, é benéfica e efetiva se implementada na rotina diária, e poderia ser obtida com a criação de protocolos e treinamento de profissionais técnicos, uma vez que a Odontologia possui um papel importante no alívio da dor e promoção da qualidade de vida (Rocha \& Ferreira., 2014). A atenção odontológica no panorama atual da saúde assume o papel de atuar nos hospitais através da promoção de saúde, prevenção de doenças bucais e redução dos danos decorrentes do internamento (Lima, et al., 2018).

Inúmeros efeitos colaterais decorrentes das terapias antineoplásicas podem ser observados na cavidade bucal, tais como: xerostomia, mucosite, cárie por radiação, osteorradionecrose, diminuição do paladar, necrose de tecido mole, neurotoxicidade, infecção bacteriana, odontogênica, das glândulas salivares e, ainda, infecções fúngicas e viróticas (Volpato, et al., 2014).

Neste estudo, as manifestações clínicas mais frequentes foram os enjoos e vômitos, o que pode ser justificado pelo fato de os quimioterápicos serem administrados sistemicamente e, por isso, causar sintomatologias como estas (Barbosa et al., 2010). A baixa incidência de manifestações bucais em crianças pode ser atribuída, em parte, à precoce idade do diagnóstico, quando há menor frequência das alterações inflamatórias e degenerativas preexistentes (Ponte, et al., 2019).

A sensação de boca seca (xerostomia) e a ausência de paladar, encontradas nos resultados deste estudo, também são encontradas na literatura atual, como no estudo de Velten, et al. (2017), onde 31,1\% das crianças apresentavam xerostomia e no estudo de Lopes, et al. (2012), no qual 45,8\% das crianças manifestavam ausência de paladar. 
Neste estudo, observou-se que a maioria dos pacientes realizava o tratamento há mais de um ano, concordando com o estudo de Lopes, et al. (2012), onde 54,2\% dos pacientes investigados estavam sob tratamento há mais de um ano. Andrade, et al. (2020) observaram que o tempo de internação variou de um a 17 dias, em 35 crianças com câncer atendidas na Clínica Pediátrica e na Unidade de Terapia Intensiva Pediátrica do Hospital Estadual Mário Covas e no ambulatório de Oncologia Pediátrica da Faculdade de Medicina do ABC (Sorocaba, SP), sendo a média de 4,7 dias, estando de acordo com os achados do nosso estudo.

Nos países em desenvolvimento, a proporção dos tumores malignos representa de 3 a $10 \%$ do total de neoplasias, sendo o diagnóstico mais comum em todo o mundo a leucemia (25 a 35\%). De acordo com o Instituto Nacional do Câncer (Brasil, 2020), os tumores mais comuns na infância são as leucemias, os tumores do sistema nervoso central e os tumores do sistema linfático. Secundariamente, podem ocorrer tumores do sistema nervoso periférico (neuroblastomas), tumores renais do tipo Wilms, retinoblastomas e osteossarcomas, tornando-os a maior causa de morte infantil por doenças não infectocontagiosas no país. Em nosso estudo, a leucemia não foi apontada como o diagnóstico mais comum, o que pode ser justificado pelo fato de que no estado do Amazonas o tratamento de neoplasias hematológicas é realizado, majoritariamente, pelo HEMOAM (Fundação Hospitalar de Hematologia e Hemoterapia do Amazonas), sendo a FCECON responsável apenas pelo tratamento dos tumores sólidos. Entretanto, o osteossarcoma e o neuroblastoma foram os tipos de câncer mais encontrados em nosso estudo.

Uma das limitações de nosso estudo foi o reduzido número de cuidadores convidados. Isso provavelmente se deva ao período de cinco meses em que fora realizada a coleta de dados. Muitas famílias se deslocam de seu local de moradia, sendo provenientes de municípios longínquos do interior do Estado do Amazonas, ou ainda, de outros Estados vizinhos, até o local de tratamento, para que este seja adequado e de qualidade e se deparam com o alto custo de locomoção, moradia e alimentação durante a sua permanência na capital, obrigando-as, quando da inexistência de vagas em casas de apoio, a retornarem ao seu local de origem.

\section{Conclusão}

Com base nos resultados da pesquisa, pôde se observar que a maior parte dos cuidadores das crianças com câncer, em tratamento, eram as mães, solteiras, que se dedicavam completamente aos cuidados dos filhos. Constatou-se falta de informação dos cuidadores sobre as práticas de higiene bucal e a dificuldade em entender a importância da saúde bucal para manutenção da saúde sistêmica, apesar de relatarem a ocorrência frequente de disgeusia e secura bucal, o que pode influenciar negativamente no sucesso do tratamento médico e até mesmo levar o paciente a óbito.

\section{Referências}

American Academy of Pediatric Dentistry. (2020). Fluoride therapy. The Reference Manual of Pediatric Dentistry. Chicago, Ill. American Academy of Pediatric Dentistry.

Andrade, A. C. M, Filipini, R., Liubartas, R., Coelho, P. G., \& Fonseca, F. L. A. (2020). Impacto físico e psicossocial na criança com câncer em tratamento: avaliando sua qualidade de vida. Revista da Faculdade de Ciências Médicas de Sorocaba, 22(1), 9-16. 10.23925/1984-4840.2020v22i1a3

Associação Brasileira de Odontpediatria. (2020). Diretrizes para procedimentos clínicos em odontopediatria. (3a ed.), Santos.

Ballestreri, R., Santo, G. W. D., Freddo, S. L., \& Lucietto, D. A. (2016). Hábitos de saúde bucal em crianças internadas no Hospital da Criança do município de Chapecó, Santa Catarina, Brasil. Revista da Faculdade de Odontologia da Universidade de Passo Fundo, $21(3), 300-305$. http://revodonto.bvsalud.org/scielo.php?script=sci_arttext\&pid=S1413-40122016000300003

Barbosa, A. M., Ribeiro, D. M., \& Caldo - Texeira, A. S. (2010). Conhecimentos e práticas em saúde bucal com crianças hospitalizadas com câncer. Ciência \& Saúde Coletiva, 15 (supl.1), 1113-1122. 10.1590/S1413-81232010000700019

Benedetti, G. M. S., Garanhani, M. L., \& Sales, C. A. (2014). O tratamento do câncer infantojuvenil: desvelando as vivências dos pais. Revista LatinoAmericana de Enfermagem, 22(3),425-431. 10.1590/0104-1169.3264.2433 
Brasil. Instituto Brasileiro de Geografia e Estatística. (2019). Pesquisa Estatística de Registro Civil. https://sidra.ibge.gov.br/tabela/4781.

Brasil. Instituto Nacional de Câncer. (2020). Câncer infantojuvenil. https://www.inca.gov.br/tipos-de-cancer/ cancer-infantojuvenil\#.

Brasil. Ministério da Saúde. Instituto Nacional do Câncer. Coordenação de Prevenção e Vigilância do Câncer. (2008). Câncer da criança e do adolescente no Brasil: dados dos registros de base populacional e de mortalidade. Esdeva. https://bvsms.saude.gov.br/bvs/publicacoes/cancer_crianca_adolescente_brasil.pdf

Finelli, L. A. C., Silva, K. J., \& Santana, M. R. (2015). Percepção da mãe quanto às consequências que o câncer do filho traz ao relacionamento conjugal. Revista Brasileira de Pesquisa em Ciências da Saúde, 2(1), 18-21. http://revistas.icesp.br/index.php/RBPeCS/article/viewFile/27/19

Floriano, D. F., Ribeiro, P. F. A., Maragno, A. C., Rossi, K., \& Simões, P. W. T. A (2017). Complicações orais em pacientes tratados com radioterapia ou quimioterapia em um hospital de Santa Catarina. Revista de Odontologia da Universidade da Cidade de São Paulo, 29 (3), 230-236. 10.26843/ro_unicidv2932017p230-236

Gandhi, K., Datta, G., Ahuja, S., Saxena, T., \& Datta, A. G. (2017). Prevalence of oral complications occurring in a population of pediatric câncer patients receiving chemotherapy. International Journal of Clinical Pediatric Dentistry, 10(2), 166-71. 10.5005/iD-iournals-10005-1428

Grado, G. F., Ehlinger, V., Godeau, E., Arnaud C., Nabet, C., Benkirane-Jessel, N., Musset, A. M., \& Offner, D. (2021). Changes in tooth brushing frequency and its associated factors from 2006 to 2014 among French adolescents: Results from three repeated cross sectional HBSC studies. PLOS ONE, 16(3), e0249129. 10.1371/journal.pone.0249129.

Huesca, I. M., Vargas, E. P., \& Da Cruz, M.M.(2018). Proteção social brasileira e demandas no tratamento oncológico infanto-juvenil. Ciência \& Saúde Coletiva, 23(11), 3965-3978. 10.1590/1413-812320182311.26932016

International Association of Paediatric Dentistry (IAPD) foundational articles and consensus recommendations. (2020). Use of fluoride for caries prevention. http://www.iapdworld.org/03_use-of-fluoride-for-caries-prevention.

Kumar, S., Tadakamadla, J., \& Johnson, N.W. (2016). Effect of toothbrushing frequency on incidence and increment of dental caries: a systematic review and meta-analysis. Journal of Dental Research, 95(11), 1230-1236. 10.1177/0022034516655315.

Lima, L. T., Giffoni, T., Franzin, L., Matsuura, E., Progiante, P., \& Goya, S. (2016). Odontologia hospitalar: competência do cirurgião-dentista. Revista UNINGÁ Review, 28(3). http://revista.uninga.br/index.php/uningareviews/article/view/1880

Lopes, I. A., Nogueira, D. N., \& Lopes, I. A. (2012). Manifestações orais decorrentes da quimioterapia em crianças de um centro de tratamento oncológico. Pesquisa Brasileira de Odontopediatria e Clinica Integrada, 12(1), 113-119. 10.4034/PBOCI.2012.121.18

Peres, P., Queiroz, A. M., Moreira, M. R., Faquim, J. P. S., \& Ferrari, M. A. C. M. (2013). Odontopediatria aplicada ao câncer infantil - manifestações clínicas e protocolos de atendimento. JMPHC | Journal of Management \& Primary Health Care, 4(3), 191-199. 10.14295/jmphc.v4i3.188

Pinheiro, I. S. P., Guerra, B. C. O., Silva, L. R., Pacheco, P. Q. C., Monnerat, I. C., Teixeira, S. V., Macedo, E. C., \& Veras, R. C. (2021). Impactos na saúde da mulher mãe cuidadora de criança com câncer. Research, Society and Development, 10(1), e31510111828. 10.33448/rsd-v10i1.11828

Ponte, Y. O., Ximenes, R. D. A., Vasconcelos, A. A., \& Girão, D. C. (2019). Saúde bucal em crianças com câncer: conhecimentos e práticas dos cuidadores. Revista da Faculdade de Odontologia da Universidade de Passo Fundo, 24(2),183-191. 10.5335/rfo.v24i2.10433

Rocha, A. L., \& Ferreira, E. F. (2014). Odontologia hospitalar: a atuação do cirurgião dentista em equipe multiprofissional na atenção terciária. Arquivos em Odontologia, 50(4),154-60. http://revodonto.bvsalud.org/scielo.php?script=sci_arttext\&pid=S1516-09392014000400001

Rottini, B. K., Lima, T. A., \& Guerra, L. F. C. (2019). Percepção dos pacientes oncológicos, sob quimioterapia, quanto às complicações orais advindas do tratamento antineoplásico em um hospital do sudoeste paranaense. Revista UNINGÁ, 56 (S5), 23-36. http://revista.uninga.br/index.php/uninga/article/view/2791

Sommerfeld, C. E., Calmon, C. M. S., Sperandio, F., Machado, A., \& Beltrame, T. S. (2011) Qualidade de vida de crianças em tratamento clínico de leucemia. Revista Brasília Médica, 48(2),129-137. https://pesquisa.bvsalud.org/portal/resource/pt/lil-603918

Velten, D. B., Zandonade, E., \& Monteiro de Barros Miotto, M.H. (2017). Prevalence of oral manifestations in children and adolescents with cancer submitted to chemotherapy. BMC Oral Health, 16 (1), 107. 10.1186/s12903-016-0300-2.

Volpato, S., Pasinato, F., Gallon, A., \& Tomasi, P. Z. (2014). Oncologia e tratamento odontológico: uma revisão. Ação Odonto, 2(1), 72-82. https://portalperiodicos.unoesc.edu.br/acaodonto/article/view/4901 